\title{
FREE INVERSE SEMIGROUPS
}

\author{
H. E. SCHEIBLICH
}

\begin{abstract}
At least three authors have offered proofs of the existence of a free inverse semigroup, but without describing its structure. This paper shows that if $X$ is a nonempty set, $G$ is the group on $X$, and $E$ is a certain subsemilattice of the power set of $G$, then a certain collection of principal ideal isomorphisms of $E$ is a free inverse semigroup on $X$.
\end{abstract}

I. Introduction. The first to ascertain the existence of a free inverse semigroup seems to have been V. V. Vagner [5]. Subsequent existence proofs have been offered in [3], and in [2]. The purpose of this paper is to provide a characterization of that semigroup and thus shed some light on its structure.

Terminology will be that of Clifford and Preston [1]. In addition, a basic knowledge of inverse semigroups and symmetric inverse semigroups is assumed.

If $X$ is a nonempty set, a free inverse semigroup on $X$ is a pair $(I, f)$ such that (i) $I$ is an inverse semigroup, (ii) $f: X \rightarrow I$, and (iii) if $S$ is any inverse semigroup and $g: X \rightarrow S$, then there exists a unique homomorphism $h: I \rightarrow S$ such that $f h=g$. Definitions of free group and free semigroup are stated similarly.

Throughout, details of induction proofs are omitted as are other proofs which seem particularly simple.

II. The free inverse semigroup on $X$. Let $X$ be a nonempty set. Let $X^{-1}$ be a set disjoint from $X$ such that $|X|=\left|X^{-1}\right|$. Let $x \rightarrow x^{-1}$ for each $x \in X$ be a bijection of $X$ onto $X^{-1}$. The union of this map with its inverse, $x \leftrightarrow x^{-1}$, is then a bijection of $Y=X \cup X^{-1}$.

Presented to the Society, January 18, 1972; received by the editors August 12, 1971. AMS (MOS) subject classifications (1970). Primary 20M10; Secondary 20M05, $20 \mathrm{M} 20$.

Key words and phrases. Free inverse semigroup.

(c) American Mathematical Society 1973 
Let $F$ be the free semigroup on $Y$, i.e., $F$ is the set of all finite sequences of elements of $Y$ with products defined by juxtaposition. Let $R=$ $\left\{w \in F: w(n+1) \neq(w(n))^{-1}\right.$ for all $\left.n\right\}$. Using the appropriate multiplication . on $R^{1}, G=R^{1}$ is then the free group on $X$ [4]. The relation

$$
\leqq=\left\{(w, v) \in R \times R: w=v u \text { for some } u \in R^{1}\right\}
$$

is a partial order for $R$. For each $w \in R$, let $w \uparrow=\{v \in R: w \leqq v\}$.

Now let $E=\{A \subseteq R: A \neq \square, A$ is finite, and if $w \in A$, then $w \uparrow \subseteq A\}$. The relation $\supseteq$ (contains) is a partial order for $E$. Further, if $A, B \in E$, then $A \cup B \in E$ and $A \cup B$ is the greatest lower bound of $\{A, B\}$. Thus $(E, \cup)$ is a semilattice. Also if $A \in E$, then $E A$, the principal ideal generated by $A$, is $\{B \in E: B \supseteq A\}$.

For each $x \in X$, let $\bar{x}: G \rightarrow G$ by

$$
\begin{aligned}
w \bar{x} & =1 & & \text { if } w=1 \\
& =x^{-1} & & \text { if } w=x \\
& =x^{-1} \cdot w & & \text { otherwise }
\end{aligned}
$$

Then for each $x \in X, \bar{x} \in \operatorname{Per}(G)$, the permutation group on $G$, and so the map $x \rightarrow \bar{x}$ extends uniquely to a homomorphism of $G$ into $\operatorname{Per}(G)$. Of course, $\overline{1}$ is the identity map of $G$ and if $x \in X$, then

$$
\begin{aligned}
w \bar{x}^{-1} & =1 & & \text { if } w=1, \\
& =x & & \text { if } w=x^{-1} \\
& =x \cdot w & & \text { otherwise. }
\end{aligned}
$$

Assume that $w \in G$. Then $\bar{w}$ may be considered to be a permutation of Pow $(G)$, the power set of $G$, where $A \bar{w}=\{v \bar{w}: v \in A\}$. Now let $A \in E$, and let $w \in A^{1}$. Then $\bar{w} \mid E A$, the restriction of $\bar{w}$ to $E A$, is an isomorphism of $E A$ onto $E(A \bar{w})$. Thus $\bar{w} \mid E A \in \mathscr{I}_{E}$, the symmetric inverse semigroup on $E$.

Lemma 2.1. Suppose that $A \in E ; w, v \in A^{1}$; and $\bar{w}|E A=\bar{v}| E A$. Then $w=v$.

Proof. Since $\bar{w}, \bar{v}$ preserve order in $E$, it follows that $A \bar{w}=A \bar{v}$. Suppose $w \neq v$. If $v=1$, then $A=A \bar{w}$ and $w \neq 1$. If $w, v \neq 1$, then assume, without loss of generality, that $v(n) \neq w(m)$ where $|v|=n$ and $|w|=m$. Then $A=A \bar{w} \bar{v}^{-1}$. But $v(1) \in A$ and so $(u) \bar{w} \bar{v}^{-1}=v(1)$ for some $u \in A$. But $\left(w v^{-1}\right)\left(\bar{w} \bar{v}^{-1}\right)=v(1)$ and so $w v^{-1}=u \in A$. In any event, $A=A \bar{u}$ for some $u \in A$ with $|u|=l \geqq 1$.

Assume first that $u(1) \neq u(l)^{-1}$. From $s \bar{u}=u$ for some $s \in A$ and $(u u) \bar{u}=u$ 
follows that $u u \in A$. By induction, $u^{n} \in A$ for all $n$, a contradiction since $A$ is finite.

Assume now that $u(1)=u(l)^{-1}$ and let $k$ be maximal with respect to $u(j)=u(l+1-j)^{-1}$ for all $j$ with $1 \leqq j \leqq k$. Then $u=p q p^{-1}$ where $|p|=k$, $|q|=l-2 k \geqq 1$, and $q(1) \neq q(l-2 k)$. Then from $\left(p q q p^{-1}\right) \bar{u}=u$ and induction follows that $p q^{n} p^{-1} \in A$ for all $n$, again a contradiction.

Lemma 2.2. Let $A \in E$ and $w \in A$. Then $A \bar{w} \in E$ and $w^{-1} \in A \bar{w}$.

Proof. Certainly $A \bar{w} \neq \square$ and $A \bar{w}$ is finite. Suppose that $p \in A \bar{w}$ and $p \leqq q$. Then $p=b \bar{w}$ for some $b \in A$. Let $|b|=m,|w|=n$, and let $k$ be maximal with respect to $b(j)=w(j)$ whenever $1 \leqq j \leqq k$.

(1) Assume first that $k=m \leqq n$. Then $p=b \bar{w}=w(n)^{-1} \cdots w(k)^{-1}$. Thus $q=w(n)^{-1} \cdots w(i)^{-1}$ for some $i$ where $k \leqq i \leqq n$. But $u=w(1) \cdots w(i) \in$ $w \uparrow \subseteq A$ and $q=u \bar{w} \in A \bar{w}$.

(2) Assume that $k=n<m$. Then $p=b \bar{w}=b(k+1) \cdots b(m)$ and so $q=b(k+1) \cdots b(l)$ where $k+1 \leqq l \leqq m$. But $u=b(1) \cdots b(l) \in b \uparrow \subseteq A$ and $q=u \bar{w} \in A \bar{w}$.

(3) Assume now that $0 \leqq k<m, n$. Then

$$
p=b \bar{w}=w(n)^{-1} \cdots w(k+1)^{-1} b(k+1) \cdots b(m)
$$

and so either $q=w(n)^{-1} \cdots w(i)^{-1}$ where $k+1 \leqq i \leqq n$ or

$$
q=w(n)^{-1} \cdots w(k+1)^{-1} b(k+1) \cdots b(l)
$$

where $k+1 \leqq l \leqq m$. In the first case $u=w(1) \cdots w(i) \in w \uparrow \subseteq A$ and $q=$ $u \bar{w} \in A \bar{w}$. In the latter case $u=b(1) \cdots b(l) \in b \uparrow \subseteq A$ and $q=u \bar{w} \in A \bar{w}$.

Finally, $w(1) \in A$ and hence $(w(1)) \bar{w}=w^{-1} \in A \bar{w}$.

Lemma 2.3. Let $A, B \in E, w \in A^{1}, v \in B^{1}$. Then $A \cup B \bar{w}^{-1} \in E$ and $w \cdot v \in\left(A \cup B \bar{w}^{-1}\right)^{1}$.

Proof. Suppose that $p \in A \cup B \bar{w}^{-1}$ and $p \leqq q$. If $p \in A$, then $q \in A \cup$ $B \bar{w}^{-1}$. Suppose $p \in B \bar{w}^{-1}$. Then $p=b \bar{w}^{-1}$ for some $b \in B$. Let $|b|=m,|w|=n$, and let $k$ be maximal with respect to $b(j)=w^{-1}(j)$ whenever $1 \leqq j \leqq k$.

(1) Assume that $k=m \leqq n$. Then

$$
\begin{aligned}
p=b \bar{w}^{-1} & =b(1) \cdots b(m)\left[\bar{w}(n)^{-1} \cdots \bar{w}(n-m+1)^{-1} \cdots \bar{w}(1)^{-1}\right] \\
& =w(1) \cdots w(n-m+1) .
\end{aligned}
$$

Thus $q \in w \uparrow \subseteq A \subseteq A \cup B \bar{w}^{-1}$.

(2) Assume that $k=n<m$. Then

$$
\begin{aligned}
p=b \bar{w}^{-1} & =b(1) \cdots b(n) \cdots b(m)\left[\bar{w}(n)^{-1} \cdots \bar{w}(1)^{-1}\right] \\
& =b(n+1) \cdots b(m) .
\end{aligned}
$$


Thus $q=b(n+1) \cdots b(i)$ where $n+1 \leqq i \leqq m$. But $u=b(1) \cdots b(i) \in b \uparrow \subseteq B$ and so $q=u \bar{w}^{-1} \in B \bar{w}^{-1} \subseteq A \cup B \bar{w}^{-1}$.

(3) Assume that $0 \leqq k<m, n$. Then

$$
\begin{aligned}
p=b \bar{w}^{-1}= & {[b(1) \cdots b(k) \cdots b(m)] } \\
& \times\left[\bar{w}(n)^{-1} \cdots \bar{w}(n-k+1)^{-1} \cdots \bar{w}(1)^{-1}\right] \\
= & w(1) \cdots w(n-k) b(k+1) \cdots b(m) .
\end{aligned}
$$

Thus $q \in w \uparrow \subseteq A \subseteq A \cup B \bar{w}^{-1}$ or $q=w(1) \cdots w(n-k) b(k+1) \cdots b(i)$ where $k+1 \leqq i \leqq m$. In the latter case $u=b(1) \cdots b(i) \in b \uparrow \subseteq B$ and $q=u \bar{w}^{-1} \in$ $B \bar{w}^{-1} \subseteq A \cup B \bar{w}^{-1}$.

The argument that $w \cdot v \in\left(A \cup B \bar{w}^{-1}\right)^{1}$ is similar.

Let $I=\left\{(A, w) \in E \times G: w \in A^{1}\right\}$. According to Lemma 2.1, $(A, w) \rightarrow$ $\bar{w} \mid E A$ is an injection of $I$ into $\mathscr{I}_{E}$ and so $I$ will be considered as a subset of $\mathscr{I}_{E}$. Thus $(A, w)^{-1}=\left(A \bar{w}, w^{-1}\right) \in I$ by Lemma 2.2. Further, if $(A, w)(B, v) \in I$, then the domain of $(A, w)(B, v)$ is $(E(A \bar{w}) \cap E B) \bar{w}^{-1}=$ $(E(A \bar{w} \cup B)) \bar{w}^{-1}=E\left(A \cup B \bar{w}^{-1}\right)$. Also $\bar{w} \bar{v}=(w \cdot v)^{-}$. Hence $(A, w)(B, v)=$ $\left(A \cup B \bar{w}^{-1}, w \cdot v\right) \in I$ by Lemma 2.3. Thus $I$ is an inverse subsemigroup of $\mathscr{I}_{E}$. Let $f: X \rightarrow I$ by $x f=(\{x\}, x)$.

THEOREM 2.4. $(I, f)$ is a free inverse semigroup on $X$.

Proof. Let $S$ be an inverse semigroup and let $g: X \rightarrow S$. Then $g$ extends to a map $g^{\prime}: Y \rightarrow S$ by $\left(x^{-1}\right) g^{\prime}=(x g)^{-1}$ for each $x \in X$. Since $F$ is a free semigroup on $Y$, and since $Y$ may be considered to be a subset of $F$, there exists a unique homomorphism $h^{\prime}: F \rightarrow S$ such that $h^{\prime} \mid Y=g^{\prime}$. When $w \in F$, then $w h^{\prime}$ will be denoted by $(w)$. Thus if $w=w(1) \cdots w(n) \in R$, then $(w)=(w(1)) \cdots(w(n))$. When no confusion seems possible, $(w)$ will be denoted more simply by $w$.

Let $K \subseteq S$ with $K$ finite. If $K=\square$, then $[K]$ will mean $1 \in S^{\mathbf{1}}$. If $K \neq \square$, then $[K]$ will mean $\prod\left\{a a^{-1}: a \in K\right\}$. Notice that if $L \subseteq S$ with $L$ finite, and $r, s \in S$, then $[K][L]=[K \cup L], r[K] r^{-1}=[r K], r\left[r^{-1} K\right]=[K] r, \quad[r K] r=$ $r[K]$, and $[r, r s]=[r s]$.

Now let $(A, w) \in I$. If $w=1$, let $(A, w)^{0}=\{(u): u \in A\} \subseteq S$. If $w \neq 1$, let $(A, w)^{0}=\{(u): u \in A, u(1) \neq w(1)\}$. For each $i$ such that $1 \leqq i<|w|$, let $(A, w)^{i}=\left\{(u): u \in R, \quad\left(\prod_{j=1}^{i} w(j)\right) u \in A\right.$, and $\left.u(1) \neq w(i+1)\right\}$, and let $(A, w)^{|w|}=\{(u): u \in R, w u \in A\}$. If $0 \leqq i \leqq|w|$, let $[A, w]^{i}=\left[(A, w)^{i}\right]$. Define $h: I \rightarrow S$ by

$$
(A, w) h=[A, w]^{0} \prod_{j=1}^{|w|}(w(j))[A, w]^{j} .
$$

It follows immediately that $g=f h$. The argument that $h$ is indeed a homomorphism will be accomplished as a sequence of lemmas. Let $(A, w)$, $(B, v) \in I$. 
LeMma 2.5. Assume $w=1$. If $v=1$, then

$$
(A, 1) h(B, 1) h=[A, 1]^{0}[B, 1]^{0}=[A \cup B, 1]^{0}=[(A, 1)(B, 1)] h .
$$

Assume $|v| \geqq 1$. Then

$$
\begin{aligned}
(A, 1) h(B, v) h= & {[A, 1]^{0}[B, v]^{0} \prod_{j=1}^{|v|}(v(j))[B, v]^{j} } \\
= & {[(u): u \in A, u(1) \neq v(1)] } \\
& \cdot[B, v]^{0}[(u): u \in A, u(1)=v(1)](v(1))[B, v]^{1} \\
& \cdot \prod_{j=2}^{|v|}(v(j))[B, v]^{j} \\
= & {[A \cup B, v]^{0}(v(1))[(u): u \in R, v(1) u \in A, u(1) \neq v(2)][B, v]^{1} } \\
& \cdot[(u): u \in R, v(1) u \in A, u(1)=v(2)] \prod_{j=2}^{|v|}(v(j))[B, v]^{j} \\
= & \cdots=[A \cup B, v]^{0} \prod_{j=1}^{|v|}(v(j))[A \cup B, v]^{j}=[(A, 1)(B, v)] h .
\end{aligned}
$$

Lemma 2.6. Assume that $|w|=1$, say $w=x \in Y$.

(1) Suppose $v=1$. Then

$$
\begin{aligned}
(A, x) h(B, 1) h & =[A, x]^{0} x[A, x]^{1}[B, 1]^{0} \\
& =[A, x]^{0} x\left[u \in B: u(1)=x^{-1}\right][A, x]^{1}\left[u \in B: u(1) \neq x^{-1}\right] \\
& =[A, x]^{0}\left[u \in R: x^{-1} u \in B\right] x[A, x]^{1}\left[u \in B: u(1) \neq x^{-1}\right] \\
& =\left[A \cup B \bar{x}^{-1}, x\right]^{0} x\left[A \cup B \bar{x}^{-1}, x\right]^{1}=[(A, x)(B, 1)] h .
\end{aligned}
$$

(2) Suppose that $|v| \geqq 1$ and $v(1)=x^{-1}$. Let $x \cdot v=b \in G$. Then

$$
\begin{aligned}
(A, x) h(B, v) h= & {[A, x]^{0} x[A, x]^{1}[B, v]^{0} x^{-1}[B, v]^{1} \prod_{j=2}^{|v|} v(j)[B, v]^{j} } \\
= & {[A, x]^{0}\left[x(A, x)^{1}\right]\left[x(B, v)^{0}\right][B, v]^{1} \prod_{j=2}^{|v|} v(j)[B, v]^{j} } \\
= & {[u \in A: u(1) \neq x, v(2)]\left[x(A, x)^{1}\right]\left[x(B, v)^{0}\right] } \\
& \cdot[B, v]^{1}[u \in A: u(1)=v(2)] \prod_{j=2}^{|v|} v(j)[B, v]^{j} \\
= & {\left[A \cup B \bar{x}^{-1}, b\right]^{0} v(2)[u \in R: v(2) u \in A, u(1) \neq v(3)][B, v]^{2} } \\
& \cdot[u \in R: v(2) u \in A, u(1)=v(3)] \prod_{j=3}^{|v|} v(j)[B, v]^{j}=\cdots \\
= & {\left[A \cup B \bar{x}^{-1}, b\right]^{0} \prod_{j=1}^{|b|} b(j)\left[A \cup B \bar{x}^{-1}, b\right]^{j} } \\
= & {[(A, x)(B, v)] h . }
\end{aligned}
$$


(3) Suppose that $|v| \geqq 1$ and $v(1) \neq x^{-1}$. Then

$$
\begin{aligned}
(A, x) h(B, v) h= & {[A, x]^{0} x[A, x]^{1}[B, v]^{0} \prod_{j=1}^{|v|} v(j)[B, v]^{j} } \\
= & {[A, x]^{0} x\left[u \in(B, v)^{0}: u(1)=x^{-1}\right]\left[u \in(A, x)^{1}: u(1) \neq v(1)\right] } \\
& \cdot\left[u \in(B, v)^{0}: u(1) \neq x^{-1}\right]\left[u \in(A, x)^{1}: u(1)=v(1)\right] \\
& \cdot \prod_{j=1}^{|v|} v(j)[B, v]^{j} \\
= & {[A, x]^{0}\left[u \in R: x^{-1} u \in(B, v)^{0}\right] x[u \in R: x u \in A, u(1) \neq v(1)] } \\
& \cdot\left[B \bar{x}^{-1}, x v\right]^{1} v(1)[u \in R: x v(1) u \in A, u(1) \neq v(2)][B, v]^{1} \\
& \cdot[u \in R: x v(1) u \in A, u(1)=v(2)] \prod_{j=2}^{|v|} v(j)[B, v]^{j} \\
= & {\left[A \cup B \bar{x}^{-1}, x v\right]^{0} x\left[A \cup B \bar{x}^{-1}, x v\right]^{1} v(1)\left[A \cup B \bar{x}^{-1}, x v\right]^{2} } \\
& \cdot[u \in R: x v(1) u \in A, u(1)=v(2)] \prod_{j=2}^{|v|} v(j)[B, v]^{j}=\cdots \\
= & {\left[A \cup B \bar{x}^{-1}, x v\right]^{0} \prod_{j=1}^{|x v|}(x v)(j)\left[A \cup B \bar{x}^{-1}, x v\right]^{j} } \\
= & {[(A, x)(B, v)] h . }
\end{aligned}
$$

LEMMA 2.7. Suppose $|w|=n \geqq 2$. Let

$$
W=\left\{u \in R:\left(\prod_{j=1}^{n-1} w(j)\right) u \in A, u(1)=w(n)\right\} .
$$

Let

$$
P=\left(A-\left(\prod_{j=1}^{n-1} w(j)\right) W, \prod_{j=1}^{n-1} w(j)\right)
$$

and let $Q=(W, w(n))$. Then $P, Q \in I,(A, w)=P Q$, and $(P h)(Q h)=(P Q) h$.

That $h$ is a homomorphism now follows by induction using Lemmas $2.5,2.6$, and 2.7. It is not difficult, using Lemma 2.7 , to see that $X f$ generates $I$. Thus $h$ is the only homomorphism from $I$ into $S$ such that $f h=g$.

\section{REFERENCES}

1. A. H. Clifford and G. B. Preston, The algebraic theory of semigroups. Vol. 1, Math. Surveys, no. 7, Amer. Math. Soc., Providence, R.I., 1961. MR 24 \#A2627.

2. Carl Eberhart and John Selden, One-parameter inverse semigroups, Trans. Amer. Math. Soc. 168 (1972), 53-66. 
3. D. B. McAlister, A homomorphism theorem for semigroups, J. London Math. Soc. 43 (1968), 355-366. MR 37 \#329.

4. A. G. Kuroš, Theory of groups. Vol. 1, GITTL, Moscow, 1953; English transl., Chelsea, New York, 1955. MR 15, 501; MR 17, 124.

5. V. V. Vagner, Generalized heaps and generalized groups with the transitive relation of compatibility, Učen. Zap. Saratov. Gos. Univ. Ser. Meh-Mat. 70 (1961), 25-39. (Russian)

Department of Mathematics, University of South Carolina, Columbia, South CAROLINA 29208 